\title{
A case of percutaneous modified Blalock-Taussig shunt downsize with multiple stent-in-graft technique
}

\author{
Roland Fiszer, Malgorzata Szkutnik, Natalia lashchuk, Jacek Bialkowski \\ Department of Congenital Heart Diseases and Paediatric Cardiology, Medical University of Silesia, Silesian Center for Heart Diseases, \\ Zabrze, Poland
}

Adv Interv Cardiol 2016; 12, 2 (44): 164-165 DOI: 10.5114/aic.2016.59367

\section{Introduction}

Pulmonary overcirculation is a common complication related to an inadequate size of a systemic to pulmonary shunt. It leads to pulmonary oedema, subsequent reduced pulmonary compliance and desaturation. There are several surgical solutions for excessively large shunts, but according to our best knowledge no interventional technique for the reduction of a systemic to pulmonary shunt size has been reported so far.

We present a complex case of percutaneous downsizing of a modified Blalock-Taussig shunt with a multiple stent-in-graft technique in a patient with single ventricle anatomy treated with staged repair.

\section{Case report}

The case concerns a 16-year-old female patient with single ventricle, pulmonary atresia and malposed great arteries after the Hemi-Fontan procedure and aortic valve homograft implantation between the right atrium and right pulmonary artery. The homograft was closed interventionally, while the left lung was separated and supplied by a Blalock Taussig shunt at the age of 7 years [1]. Nine years later, this shunt became occluded and the left lung was supplied by collaterals only. The shunt was replaced by an $8 \mathrm{~mm}$ Gore-Tex tube graft. After an initial increase in the oxygen saturation from $60 \%$ to mid $70 \%$, she developed massive left sided pleural effusions with haemodynamic compromise. It was clear that the left sided shunt was too large. So far the only therapeutic option for patients with pulmonary overcirculation due to an inadequate shunt size has been surgery. However, a seventh surgical intervention was much too risky and undesirable for the patient. The decision was made to place several bare stents into the shunt in order to reduce the internal graft lumen. The procedure was performed under general anaesthesia. An initial angiography confirmed a large $8 \mathrm{~mm}$ left shunt (Figure 1). The shunt was crossed with the JR catheter and Terumo glide wire from the right femoral artery. Then the Cook extra-stiff wire and over it the Mullins long $7 \mathrm{Fr}$ sheath were advanced into the left pulmonary artery. Four Genesis XD stents (Johnson \& Johnson, NJ, USA; two $10 \mathrm{~mm} \times 25 \mathrm{~mm}$ and two $10 \mathrm{~mm} \times 29 \mathrm{~mm}$ ) mounted on Opta Pro balloons (Cordis, NJ, USA) were implanted into the Gore-Tex tube one over another. Control angiography confirmed the proper position of the devices (Figure 2). The day after the procedure oxygen saturation rose from $45 \%$ to $60 \%$ (with no additional oxygen supply) and transthoracic echocardiogram (TTE) showed an increase in the peak gradient across the shunt from 10 to $25 \mathrm{~mm} \mathrm{Hg}$. In the next few days the patient's condition improved, with better exercise tolerance and diminished oedemas. The chest X-ray showed a well-aired left lung. She was discharged home after 2 weeks and keeps steadily improving. The 4-month follow-up showed oxygen saturation of approximately $70 \%$ and left pulmonary artery pressure of $42 / 36 / 40 \mathrm{~mm} \mathrm{Hg}$ in the last cardiac catheterization.

\section{Discussion}

The gold standard for systemic to pulmonary shunt downsizing remains surgery. Several surgical techniques have been described, e.g., shunt replacement, shunt banding and placing a reversible haemostatic clip. Solely single attempts of percutaneous vessel size reduction have been published. The most often described interventional method of vessel downsizing is the application of a silk suture at the midpoint of a covered stent mounted on a balloon [2, 3]. After the balloon inflation such a selfmade narrowing in the stent adapts to the vessel and causes increased resistance to blood flow. Stenting of

\section{Corresponding author:}

Roland Fiszer MD, PhD, Department of Congenital Heart Diseases and Paediatric Cardiology, Medical University of Silesia, Silesian Center for Heart Diseases, 9 Curie-Skłodowskiej St, 41-800 Zabrze, Poland, phone: +48 504125 057, e-mail: rfiszer@gumed.edu.pl

Received: 7.10 .2015 , accepted: 8.12.2015. 


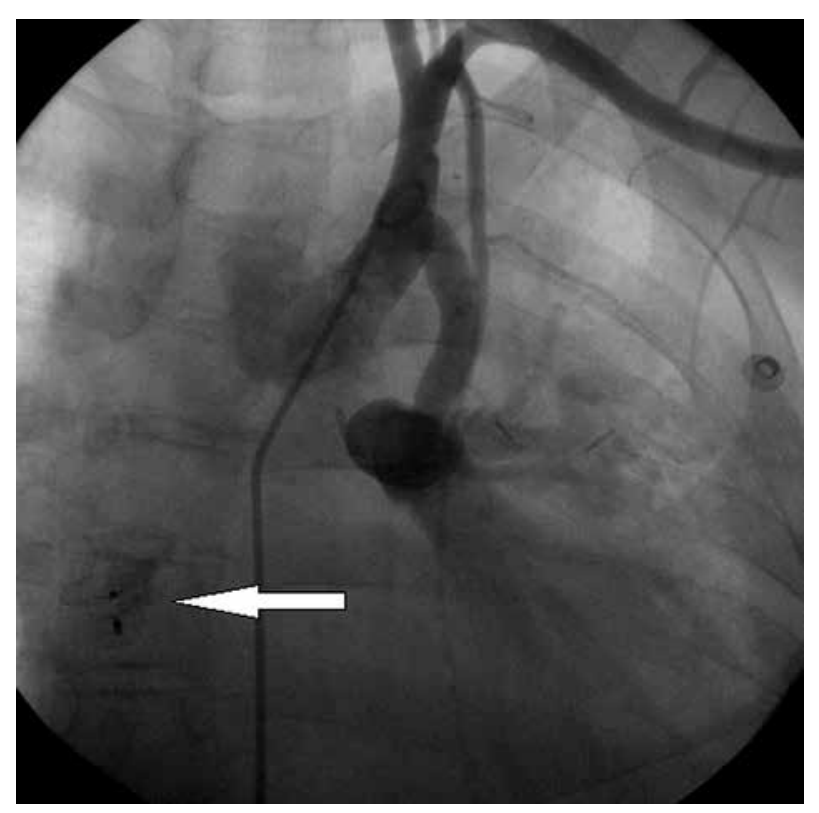

Figure 1. Angiography in PA projection - visible large (8 $\mathrm{mm}$ in diameter) shunt (Gore-tex tube) between left subclavian artery and left pulmonary artery. Arrow indicates the position of Amplatzer Duct Occluder (ADO) closing connection between right pulmonary artery and right atrium

systemic to pulmonary shunts has already been shown to be a safe and effective therapeutic option for patients with shunt stenosis or failure. Various stents were implanted in the published reports [2, 4, 5], but only in order to reduce shunt stenosis or occlusion. The main objective of our procedure was to reduce the shunt lumen by placing several stents one over another, thus increasing resistance to blood flow. Additionally, it is expected that neointimal proliferation over bare stents will result in a further reduction of approximately 0.5 to $1 \mathrm{~mm}$ of the lumen diameter with time $[3,6]$.

\section{Conflict of interest}

The authors declare no conflict of interest.

\section{References}

1. Kusa J, Szkutnik M, Skalski J, et al. Percutaneous closure of the shunt from right pulmonary artery to right atrium using Ampaltzer Duct Occluder in a child after hemi-Fontan operation. Postep Kardiol Inter 2007; 3: 113-5.

2. Krasemann T, Qureshi S. Stenting of stenosed Blalock-Taussig shunt after Norwood-I palliation for hypoplastic left heart. Heart 2007; 93: 1509.

3. Madoff DC, Wallace MJ. Reduced stents and stent-grafts for the management of encephalopathy after transjugular intrahepatic portosystemic shunt creation. Semin Intervent Radiol 2005; 22: 316-28.

4. Vaughn GR, Moore JW, Mallula KK, et al. Transcatheter stenting of the systemic-to-pulmonary artery shunt: a 7-year experience

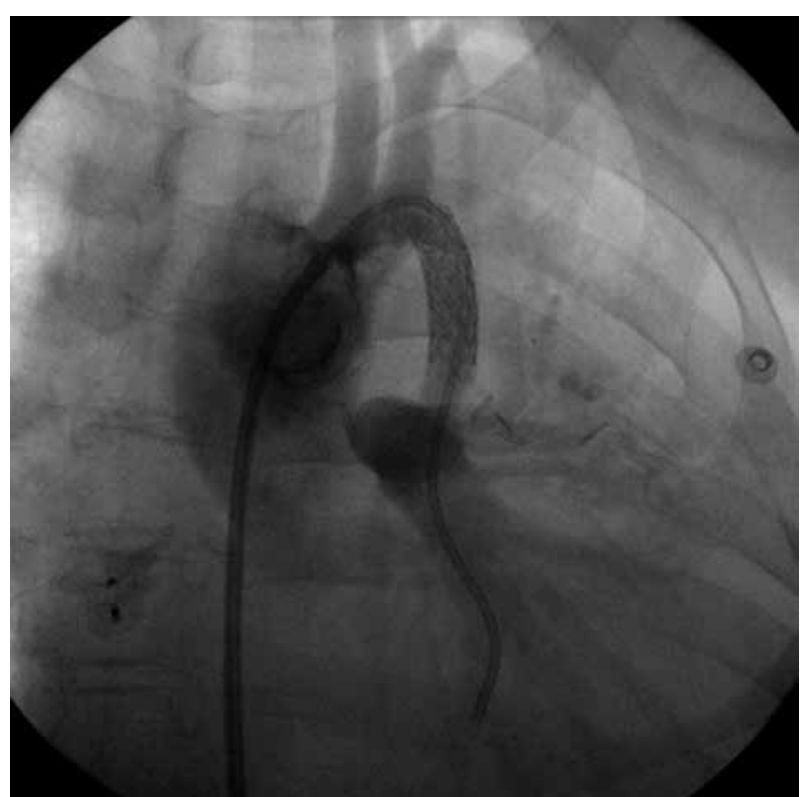

Figure 2. Angiography through the side arm of the Mullins sheath. Four stents inside the Gore-tex tube reducing the flow in the shunt are visible

from a single tertiary center. Catheter Cardiovasc Interv 2015; 86: 454-62

5. Krasemann T, Tzifa A. Stenting of modified and classical Blalock-Taussig shunt - lessons learned from seven consecutive cases. Cardiol Young 2011; 21: 430-5.

6. Nakahata Y, Tomita H. Percutaneous bilateral pulmonary artery bandingusing re-expandable covered stent: preliminary animal study. Kitasato Med J 2011; 41: 165-9. 